\title{
BMJ Open Evaluation of foot health related quality of life in individuals with foot problems by gender: a cross-sectional comparative analysis study
}

\author{
Daniel López-López, ${ }^{1}$ Ricardo Becerro-de-Bengoa-Vallejo, ${ }^{2}$ \\ Marta Elena Losa-Iglesias, ${ }^{3}$ Patricia Palomo-López, ${ }^{4}$ David Rodríguez-Sanz, ${ }^{2,5}$ \\ Juan Manuel Brandariz-Pereira, ${ }^{1}$ César Calvo-Lobo ${ }^{6}$
}

To cite: López-López D, Becerro-de-Bengoa-Vallejo R, Losa-Iglesias ME, et al. Evaluation of foot health related quality of life in individuals with foot problems by gender: a cross-sectional comparative analysis study. BMJ Open 2018;8:e023980. doi:10.1136/ bmjopen-2018-023980

- Prepublication history for this paper is available online. To view these files, please visit the journal online (http://dx.doi. org/10.1136/bmjopen-2018023980).

DL-L, RB--B-V, MEL-I, PP-L, DR$\mathrm{S}$, JMB-P and CC-L contributed equally.

Received 4 May 2018

Revised 28 August 2018

Accepted 12 September 2018

D Check for updates

(C) Author(s) (or their employer(s)) 2018. Re-use permitted under CC BY-NC. No commercial re-use. See rights and permissions. Published by BMJ.

For numbered affiliations see end of article.

Correspondence to Dr Patricia Palomo-López; patibiom@unex.es

\section{ABSTRACT}

Objective Foot problems (FP) may be considered to be a prevalent conditions in people but there is limited evidence of their effect on the quality of life (QoL) related to foot health in men and women. The aim of this study was to assess the impact of FPs on both overall foot health and QoL, stratified by gender.

Design A cross sectional study.

Setting Clinic of Podiatric Medicine and Surgery at University of A Coruña in the city of Ferrol (Spain).

Subjects The sample consisted of 1647 participants with FP; the total population of the sample had a mean age of $43.24 \pm 19.89$ years, with mean ages $44.09 \pm 21.36$ years and $42.94 \pm 19.34$ years for men and women, respectively. Methods Measurement of sociodemographic characteristics include age, sex and body mass index. The Foot Health Status Questionnaire (FHSQ) was used to evaluate the general health and foot health. Differences between groups were evaluated by means of a t-test for independent samples.

Results Women with FP showed significantly lower scores in the domains of Foot Pain, Foot Function, Footwear, General Foot Health, Physical Activity and Vigour, and there was no difference compared with men in the domains of Overall Health and Social Capacity. Conclusions Women with FP present a negative impact on QoL related to foot health as compared with men except in the domains of Overall Health and Social Capacity, which appears to be associated with the presence of foot conditions.

\section{INTRODUCTION}

Foot problems (FP) are frequently observed in the general population, with higher prevalence rates of $61.3 \%-79 \%$ reported in institutional and clinical settings. ${ }^{1-3}$ However little is known about the burden of these problems from a public health perspective and the overall burden within the general population remains unclear. ${ }^{4-6}$

Thus, FP may be one of the most frequent reasons for people seeking consultations at primary care units, and many of these
Strengths and limitations of this study

- This research presents a new framework for evaluating the impact of foot problems (FPs) on overall health and foot health.

- This method provides identification of FPs stratified by gender, related to quality of life (QoL).

- This study has been the first to show how people with FP experience a negative impact on their QoL related to foot health.

- A case-control study and a sample of people from other countries would improve the strength of this research.

- Preventative foot care is extremely important in the management of general health and foot health.

conditions may be chronic, decrease the ability to undertake activities of daily living, generate problems with balance and gait and increase the risk of falls. ${ }^{6-8}$ Further, these FPs may show an increased prevalence in women and in older age groups (>65 years), ${ }^{89}$ and present with notable health problems related to obesity, diabetes, vascular alterations, depression, risk of falls and difficulty putting on shoes, and pose to be major contributors towards the costs for foot surgery. ${ }^{10}$

In the initial approach, any FP should be considered as a specific pathology in which there are no two conditions that can be considered identical because the medical history and family history affect its evolution and prognosis. ${ }^{11}$

Despite the high prevalence of FPs in the population, their impact on overall foot health and quality of life (QoL), as stratified by gender, has not previously been studied. A meta-analysis by Thomas et al conducted on 75505 people showed that $24 \%$ of people above 45 years of age exhibited foot and ankle pain, which increased in prevalence 
with age, especially in female populations. The most frequent anatomical sites for foot pain were the toes and forefoot, which increased with age and among women. ${ }^{11}$ Furthermore, Garrow et al showed that foot disability was also associated with pain in other body parts such as the shoulder, axial skeleton, hip, upper leg and knee along with other poor general health indicators. ${ }^{12}$

According to these antecedents, and considering the existence of the attention and follow-up necessity in foot care of patients with FP, the role of the physician is important in recognising and treating the underlying condition, preventing further injury or deformities, and educating the patient about his/her disease, ${ }^{2}$ to achieve better QoL and state of well-being for people.

There is little published information on the effects of FP on QoL in the general population and especially in men and women. We hypothesised that FPs affecting QoL varied by gender. Therefore, the aim of this study was to assess the impact of FPs on both, overall foot health and QoL as determined by gender.

\section{MATERIALS AND METHODS Design and sample}

A total of 1647 patients took part in an observational study carried out at the Clinic of Podiatric Medicine and Surgery that provides treatment for diseases and disorders of the foot at the University of A Coruna in the city of Ferrol (Galicia, North-Western Spain) during the period January 2012 and June 2016. The selection of the cases subject to the research was made by a consecutive sampling technique. The ages of the people included in the study ranged from 18 years to 96 years. Disregarded and excluded cases included: patients who had experienced acute pathological fracture; previous trauma and feet surgery records; active systemic neoplastic conditions; infectious, autoimmune or neurological alterations; lack of or partial autonomy in daily activities; the refusal to sign an informed consent form or those who were incapable of understanding the instructions necessary to carry out the present study; and participants of other nationalities (non-Spanish) who did not adequately understand Spanish.

\section{Procedure}

A single trained researcher (DL-L) recorded baseline measurements. The first step included an interview about disease and clinical characteristics included age, sex and the presence of predisposing factors (diabetes, obesity, depression, vascular disease, trauma, osteoarticular pathology or participation in sports).

In the second step, patients removed their footwear and hosiery. Following this a single researcher (DL-L) assessed height and weight with the subject barefoot and wearing light clothing, and determined the body mass index (BMI). ${ }^{13}$

Then, the physician (DL-L) examined foot disorders and the structural integrity of the foot through clinical assessment, neurovascular palpation, musculoskeletal mobility and strength testing on the foot using the de Kendall test. Also, diagnosis of the FPs was assessed using ultrasonography or previous radiographic evidence, since full access to the clinical records of the patient was available, to check for any other foot pathology and systemic diseases of the patient.

Lastly, impact on the foot health and overall health was assessed and compared using the Foot Health Status Questionnaire (FHSQ). ${ }^{14} 15$ This self-administered questionnaire on health-related QoL, intended specifically for the foot, is recognised as a validated test as described in detail previously. ${ }^{16} 17$ This questionnaire consists of three sections. Section 1 has 13 questions about four foot health related subcategories: foot function, foot pain, footwear and general foot health. Section 2 includes the four subscales or domains of overall health: general health, physical function, social function and vitality.

Finally, Section 3 collects socioeconomic status, comorbidities, service utilisation and satisfaction information and their medical records.

The questionnaire does not show a global score, but rather provides an index for each subscale or domain. Consequently, the responses are obtained by a computer program (FHSQ V.1.03) which, after data processing, provides a score from 0 to 100 . A 0 score shows the worst foot health state and a 100 score reflects the best possible condition. Furthermore, outcomes using graphical images are provided by this software.

\section{Patient and public involvement}

Patients were not involved in the conceived and designed of this research. Currently, there is no plan to transfer these results to study participants.

\section{Ethical considerations}

Participation in the study was voluntary and all participants provided written informed consent before the start of the study. Human research ethical standards according to the Helsinki Declaration, the human rights and biomedicine statements of the Convention of the Council of Europe, as well as human genome and rights from the Unesco Universal Declaration and other appropriate national or institutional organisations were followed.

\section{Sample size}

The sample size was calculated using the software from Unidad de Epidemiología Clínica y Bioestadística, Complexo Hospitalario Universitario de A Coruña, Universidade da Coruña (www.fisterra.com) ${ }^{18}$ The calculations were based on the total population of the city of Ferrol, that is 69452 (http://www.ine.es/jaxiT3/Datos. htm?t=2868), 95\% CI, an $\alpha$ level of 0.05 , and a proportion of $50 \%$ and a precision of $1 \%$, a minimum final sample size of 453 participants was calculated. Assuming a dropout of $15 \%$ of participants, at least 533 participants must be studied. Finally, a total of 1647 people were included in the study. 


\section{Statistical analysis}

Demographic characteristics, including subject age, height, weight and BMI, and independent variables were summarised as mean and $\mathrm{SD}$, and maximum and minimum values. For categorical variables, the frequency and percentage were used.

The Kolmogorov-Smirnov test was used to evaluate normality, and data were determined as normally distributed if $p>0.05$. The results of these tests indicated that the data were normally distributed and that parametrical statistical tests were most appropriate. Independent Student's t-tests were performed to determine whether statistically significant differences were obtained in all variables between the two groups, that is, men and women. The $\chi^{2}$ test was used to determine whether a significant difference was shown between the observed frequencies.

FSHQ V.1.03 was used to determine QoL scores with respect to foot health. In all analyses, statistical significance was considered with a $p$ value $<0.01$ with a $99 \%$ CI.

For demonstrating the effect size of the comparisons, Cohen's d coefficient was calculated. The standard interpretation offered by Cohen and expanded by Sawilowsky ${ }^{19}$ is as follows: $0.01=$ verysmall; $0.20=$ small; $0.50=$ medium; $0.80=$ large; $1.20=$ very large; and 2.0 or higher $=$ huge.
A p value $<0.01$ with a CI of $99 \%$ was considered statistically significant for all tests (SPSS for Windows, V.20.0; SPSS, Chicago, Illinois, USA).

All analyses were carried out with a commercially available statistical software (SPSS V.19.0, Chicago, Illinois, USA).

\section{RESULTS}

A total of 1647 participants ranging in age from 18 years to 96 years completed the research course. The sample analysed included $1215(73.77 \%)$ women and $432(26.23 \%)$ men.

All variables showed a normal distribution $(\mathrm{p}>0.05)$.

Table 1 shows the clinical and sociodemographic characteristics of the informants. There were no statistically significant differences between sex and age $(\mathrm{p}=0.30)$ and sex and BMI $(p=0.48)$.

According to the WHO BMI classification, ${ }^{20} 17$ (1.03\%) participants proved to be underweight with a BMI $<18.0$; another $945(57.37 \%)$ had normal weight with values between 18.5 and 24.9; another 518 (31.45\%) showed preobesity with values between 25.0 and 29.9; another $157(9.53 \%)$ had obesity class I with values between 30 . and 34.9; another $9(0.54 \%)$ had obesity class II with values between 35.0 and 39.9 and only 1 participant $(0.06 \%)$ was classified as obesity class III with values above

Table 1 Sociodemographic and clinical characteristics of the sample population

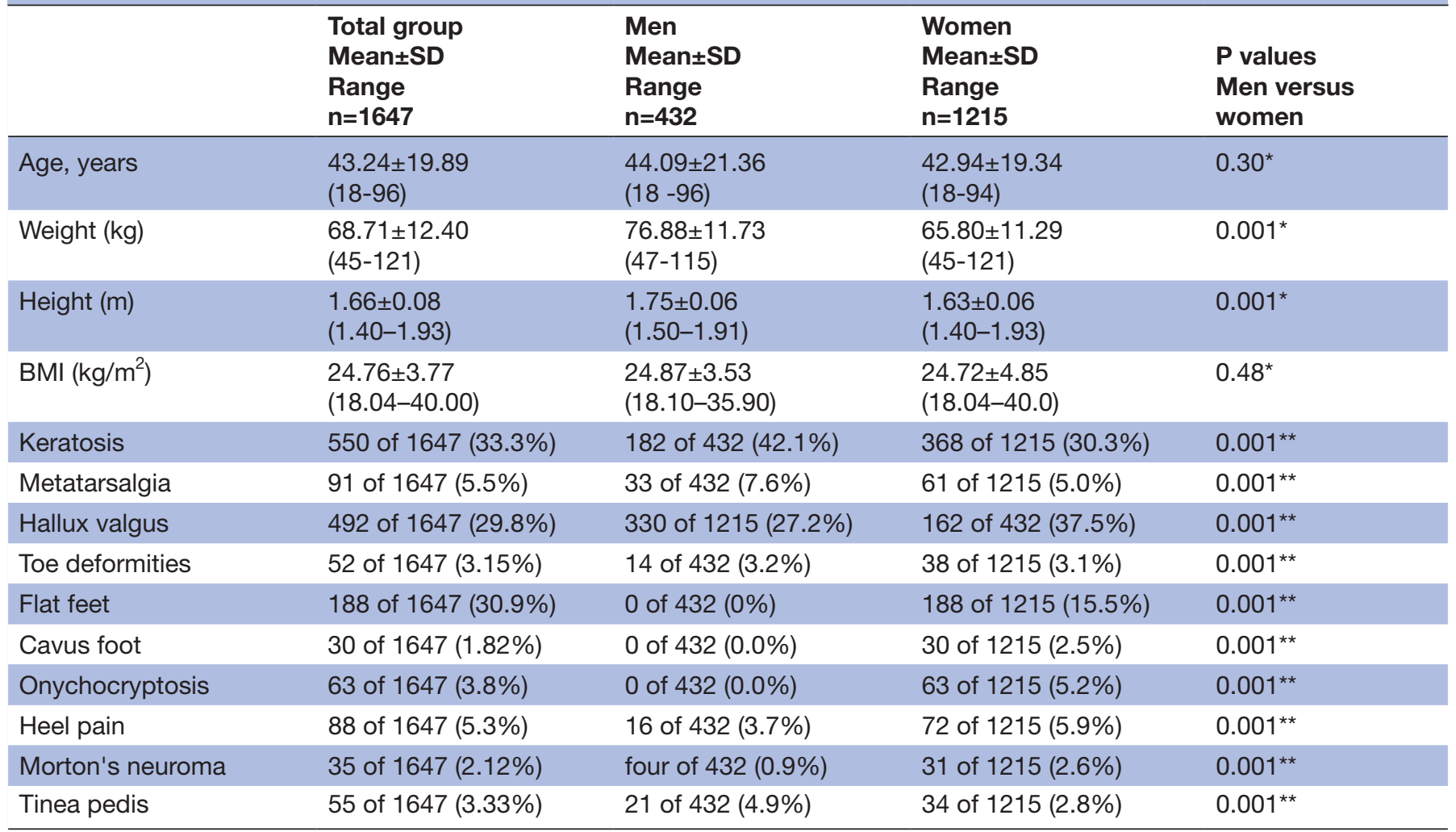

In all the analyses, $\mathrm{p}<0.01(99 \% \mathrm{Cl})$ was considered statistically significant.

*Independent Student's t-test was used.

${ }^{\star *} \chi^{2}$ test was used.

$\mathrm{BMI}$, body mass index. 
Table 2 Comparisons of FHSQ scores in the total group and gender groups

\begin{tabular}{|c|c|c|c|c|c|}
\hline & $\begin{array}{l}\text { Total group } \\
\text { Mean (SD) } \\
\text { Range (n=1647) }\end{array}$ & $\begin{array}{l}\text { Men } \\
\text { Mean (SD) } \\
\text { Range (n=432) }\end{array}$ & $\begin{array}{l}\text { Women } \\
\text { Mean (SD) } \\
\text { Range (n=1215) }\end{array}$ & $\begin{array}{l}P \text { values Men } \\
\text { versus women }\end{array}$ & $\begin{array}{l}\text { Cohen's d Effect } \\
\text { size }\end{array}$ \\
\hline Foot pain & $\begin{array}{l}74.61 \pm 21.94 \\
(0-100)\end{array}$ & $\begin{array}{l}79.11 \pm 19.51 \\
(0-100)\end{array}$ & $\begin{array}{l}73.01 \pm 22.53 \\
(0-100)\end{array}$ & $<0.001$ & 0.289 \\
\hline Foot function & $\begin{array}{l}82.19 \pm 21.17 \\
(0-100)\end{array}$ & $\begin{array}{l}85.48 \pm 18.45 \\
(0-100)\end{array}$ & $\begin{array}{l}81.03 \pm 21.94 \\
(0-100)\end{array}$ & $<0.001$ & 0.219 \\
\hline Footwear & $\begin{array}{l}50.75 \pm 30.60 \\
(0-100)\end{array}$ & $\begin{array}{l}58.58 \pm 29.31 \\
(0-100)\end{array}$ & $\begin{array}{l}47.96 \pm 30.58 \\
(0-100)\end{array}$ & $<0.001$ & 0.354 \\
\hline General foot health & $\begin{array}{l}57.35 \pm 27.44 \\
(0-100)\end{array}$ & $\begin{array}{l}64.56 \pm 25.76 \\
(0-100)\end{array}$ & $\begin{array}{l}54.79 \pm 27.57 \\
(0-100)\end{array}$ & $<0.001$ & 0.366 \\
\hline Overall health & $\begin{array}{l}66.63 \pm 23.39 \\
(0-100)\end{array}$ & $\begin{array}{l}66.88 \pm 22.31 \\
(0-100)\end{array}$ & $\begin{array}{l}66.55 \pm 23.77 \\
(0-100)\end{array}$ & 0.80 & 0.014 \\
\hline Physical activity & $\begin{array}{l}82.98 \pm 22.09 \\
(0-100)\end{array}$ & $\begin{array}{l}87.01 \pm 18.98 \\
(0-100)\end{array}$ & $\begin{array}{l}81.55 \pm 22.93 \\
(0-100)\end{array}$ & $<0.001$ & 0.259 \\
\hline Social capacity & $\begin{array}{l}80.22 \pm 23.60 \\
(0-100)\end{array}$ & $\begin{array}{l}81.51 \pm 22.72 \\
(0-100)\end{array}$ & $\begin{array}{l}79.77 \pm 23.90 \\
(0-100)\end{array}$ & 0.18 & 0.074 \\
\hline Vigour & $\begin{array}{l}55.09 \pm 21.54 \\
(0-100)\end{array}$ & $\begin{array}{l}59.90 \pm 19.68 \\
(0-100)\end{array}$ & $\begin{array}{l}53.38 \pm 21.92 \\
(0-100)\end{array}$ & $<0.001$ & 0.313 \\
\hline
\end{tabular}

In all the analyses, $\mathrm{p}<0.01$ (with a $99 \% \mathrm{Cl}$ ) was considered statistically significant. FHSQ, Foot Health Status Questionnaire.

40. Also, $100 \%(\mathrm{n}=1647)$ of the participants stated they had suffered one or more FP; this was determined in the study procedure by the physician (DL-L) and the clinical characteristics are as shown in table 1.

Moreover, the total population showed that $29.9 \%$ $(\mathrm{n}=492)$ had hallux valgus, $11.4 \% \quad(\mathrm{n}=188)$ had flat foot, $5.7 \%(\mathrm{n}=94)$ had metatarsalgia, $5.5 \%(\mathrm{n}=91)$ had keratosis, $5.3 \% \quad(\mathrm{n}=88)$ had heel pain, $3.8 \% \quad(\mathrm{n}=63)$ had onychocryptosis, $3.2 \%(\mathrm{n}=52)$ had toe deformities, $1.8 \%(\mathrm{n}=30)$ had cavus foot, $2.1 \%(\mathrm{n}=35)$ had Morton's neuroma, 55 of 1647 (3.33\%) had tinea pedis, and all of these conditions were shown to be significantly higher in women than in men $(\mathrm{p}<0.01)$.

Furthermore, $27.9 \%$ of the patients who participated in the research presented with predisposing factors including $11 \% \quad(\mathrm{n}=181)$ with vascular disease, $9.1 \%$ $(\mathrm{n}=150)$ osteoarticular pathology, 3.8\% $(\mathrm{n}=62)$ diabetes, 2.7\% $(n=45)$ obesity and $1.2 \% \quad(n=20)$ suffered from depression.

The results of FHSQ scores between men and women with FP are shown in table 2. Women with FP showed significantly lower scores in the domains of foot pain, foot function, footwear, general foot health, physical activity and vigour with $\mathrm{p}<0.001$ in all of them, and there was no difference by gender in the domains of overall health and social capacity with $\mathrm{p}>0.01$ in both.

The effect sizes are shown in table 2. A small effect size was found by gender at general foot health, footwear, vigour, foot pain, physical activity, foot function with values of $0.366,0.354,0.313,0.289,0.259$ and 0.366 , respectively. The effect sizes were very small for social capacity and overall health with values of 0.074 and 0.014 , respectively.

\section{DISCUSSION}

The purpose of this study was to assess and compare the impact of foot health in a sample of people with FPs, with the help of scores obtained with regard to foot health and health in general between men and women.

Foot care is an essential need, given the high prevalence $(100 \%)$ of people who complain of foot disorders. Also, these findings are consistent with previous epidemiological research from other authors. ${ }^{321}{ }^{22}$ Furthermore, a systematic review about foot pain concurred with our results, also recognising that the prevalence of foot pain was higher in women than in men. ${ }^{11}$ These findings are similar to ours with regard to the QoL related to foot health, where women also showed lower and worse scores than men.

Therefore, it is recommended that foot health should be managed by physicians/podiatrists well before FPs limit the individuals' ability with respect to foot function and ability to self-manage. Also, our results showed the negative impact of the QoL related to foot health in people with FP. This is consistent with the results of other studies linking QoL to other foot diseases. These studies have demonstrated that hallux valgus, calcaneal apophysitis, foot arch height, heel pain, chronic ankle instability and onychomycosis negatively affect foot function and foot health. ${ }^{23-27}$

This study has been the first to examine the impact related to foot health in a sample of people with FP between men and women.

The findings of this study need to be interpreted in the context of several inherent limitations that should be acknowledged. A sample with diverse patients from other countries and cultures would be beneficial to improve the strength of the research and may help to identify if there is a culture or location where this association does not exist 
and identify if other factors could influence the differences between men and women in relation to foot pathology and thus impact on QoL: sociodemographics, types of employment, levels of physical activity, potential impact of pregnancy and child bearing on joints, and medical health insurance. Furthermore, a case-control study could clarify the QoL differences related to foot health between subjects with and without FP, in order to stratify both groups by sex. These limitations should be addressed in future studies.

In light of these findings, further continuous research on this trend of FPs is required in order to evaluate the effectiveness of the available treatments, improve the examination routine of patients' feet by podiatrist and physicians, reduce the burden of these foot conditions and improve the QoL in the general population and specifically in women who present a worse QoL related to foot health.

When comparing the domains of social capacity and overall health between men and women to find out the effect size, we found that the means of two groups don't differ by $0.2 \mathrm{SD}$, so the difference is very small or trivial. When comparing general foot health, footwear, vigour, foot pain, physical activity and foot function dimensions, the effect sizes were small, even though they were statistically significant.

Further work is necessary to better understand the extent and type of unmet needs and how patients, especially women, presenting with symptoms of disabling foot pain should best be managed.

\section{CONCLUSIONS}

Women with FPs present a negative impact on the QoL related to foot health with respect to men, except in the domains of overall health and social capacity, which appear to be associated with the presence of foot conditions.

\section{Author affiliations}

${ }^{1}$ Research, Health and Podiatry Unit, Department of Health Sciences, Faculty of Nursing and Podiatry, Universidade da Coruña, Ferrol, A Coruña, Spain

${ }^{2}$ Facultad de Enfermería, Fisioterapia y Podología, Universidad Complutense de Madrid, Madrid, Spain

${ }^{3}$ Faculty of Health Sciences, Universidad Rey Juan Carlos, Alcorcón, Madrid, Spain ${ }^{4}$ University Center of Plasencia, Universidad de Extremadura, Plasencia, Cáceres, Spain

${ }^{5}$ Faculty of Sports Sciences, European University of Madrid, Villaviciosa de Odón, Madrid, Spain

${ }^{6}$ Nursing and Physical Therapy Department, Faculty of Health Sciences, Universidad de León, Ponferrada, León, Spain

Acknowledgements The authors thank the persons that participated in this research.

Contributors DL-L, MEL-I, RB-de-B-V, PP-L, DR-S, CC-L, JMB-P conceived and designed the study. DL-L, MEL-I and RB-de-B-V analysed and interpreted the data. DL-L, MEL-I, RB-de-B-V, PP-L, DR-S, CC-L, JMB-P drafted the manuscript and all authors contributed to the revisions of the manuscript and approved the final draft for submission.

Funding The authors have not declared a specific grant for this research from any funding agency in the public, commercial or not-for-profit sectors.

Competing interests None declared.

Patient consent Obtained.

Ethics approval Research and Ethics Committee of A Coruña University (Spain), file number CE 38/2012.
Provenance and peer review Not commissioned; externally peer reviewed.

Data sharing statement The data set supporting the conclusions of this article is available with DL-L at daniellopez@udc.gal.

Open access This is an open access article distributed in accordance with the Creative Commons Attribution Non Commercial (CC BY-NC 4.0) license, which permits others to distribute, remix, adapt, build upon this work non-commercially, and license their derivative works on different terms, provided the original work is properly cited, appropriate credit is given, any changes made indicated, and the use is non-commercial. See: http://creativecommons.org/licenses/by-nc/4.0/.

\section{REFERENCES}

1. Pita-Fernandez S, González-Martín C, Seoane-Pillado T, et al. Podiatric medical abnormalities in a random population sample 40 years or older in Spain. J Am Podiatr Med Assoc 2014;104:574-82.

2. López-López D, García-Mira R, Palomo-López P, et al. Attitude and knowledge about foot health: a spanish view. Rev Lat Am Enfermagem 2017;25:e2855.

3. Graham AS, Stephenson J, Williams AE. A survey of people with foot problems related to rheumatoid arthritis and their educational needs. J Foot Ankle Res 2017;10:12.

4. Bowling A, Grundy E. Activities of daily living: changes in functional ability in three samples of elderly and very elderly people. Age Ageing 1997;26:107-14.

5. Barr EL, Browning C, Lord SR, et al. Foot and leg problems are important determinants of functional status in community dwelling older people. Disabil Rehabil 2005;27:917-23.

6. Menz HB, Morris ME. Footwear characteristics and foot problems in older people. Gerontology 2005;51:346-51.

7. Nix S, Smith M, Vicenzino B. Prevalence of hallux valgus in the general population: a systematic review and meta-analysis. J Foot Ankle Res 2010;3:21.

8. Rodríguez-Sanz D, Tovaruela-Carrión N, López-López D, et al. Foot disorders in the elderly: A mini-review. Dis Mon 2018;64.

9. Benvenuti F, Ferrucci L, Guralnik JM, et al. Foot pain and disability in older persons: an epidemiologic survey. J Am Geriatr Soc 1995;43:479-84.

10. Woolf AD, Akesson K. Primer: history and examination in the assessment of musculoskeletal problems. Nat Clin Pract Rheumatol 2008;4:26-33.

11. Thomas MJ, Roddy E, Zhang W, et al. The population prevalence of foot and ankle pain in middle and old age: a systematic review. Pain 2011;152:2870-80.

12. Garrow AP, Silman AJ, Macfarlane GJ. The Cheshire Foot Pain and Disability Survey: a population survey assessing prevalence and associations. Pain 2004;110(1-2):378-84.

13. Centers of disease control, Body mass index: Considerations for practitioners. Cdc. 2011;4 http://scholar.google.com/scholar?hl= en\&btnG=Search\&q=intitle:Body+Mass+Index+:+Considerations+ for+Practitioners\#3\%5Cnhttp://scholar.google.com/scholar?hl=en\& bth $\mathrm{G}=$ Search\&q=intitle:Body+mass+index:+Considerations+for+ practitioners\#3.

14. Bennett PJ, Patterson C, Dunne MP. Health-related quality of life following podiatric surgery. J Am Podiatr Med Assoc 2001;91:164-73.

15. Bennett PJ, Patterson C, Wearing S, et al. Development and validation of a questionnaire designed to measure foot-health status. J Am Podiatr Med Assoc 1998;88:419-28.

16. Burzykowski T, Molenberghs G, Abeck D, et al. High prevalence of foot diseases in Europe: results of the Achilles Project. Mycoses 2003;46(11-12):496-505.

17. Hagedorn TJ, Dufour AB, Riskowski JL, et al. Foot disorders, foot posture, and foot function: the Framingham foot study. PLoS One 2013;8:e74364.

18. Pita Fernández S. Determinación del tamaño muestral. Cad Aten primaria 1996;3:138-52.

19. Sawilowsky S. New effect size rules of thumb. Mod Appl Stat Methods 2009;8:467-74.

20. Body mass index - BMI. 2018. http://www.euro.who.int/en/healthtopics/disease-prevention/nutrition/a-healthy-lifestyle/body-massindex-bmi

21. Irving DB, Cook JL, Young MA, et al. Impact of chronic plantar heel pain on health-related quality of life. J Am Podiatr Med Assoc 2008;98:283-9.

22. James AM, Williams CM, Haines TP. Health related quality of life of children with calcaneal apophysitis: child \& parent perceptions. Health Qual Life Outcomes 2016;14:1-7. 
23. López López D, Bouza Prego ML, Requeijo Constenla A, et al. The impact of foot arch height on quality of life in 6-12 year olds. Colomb Med 2014;45:168-72.

24. Palomo-López P, Becerro-de-Bengoa-Vallejo R, Losa-Iglesias ME, et al. Impact of Hallux Valgus related of quality of life in Women. Int Wound J 2017;14:782-5.

25. Tennvall GR, Norlin JM, Malmberg I, et al. Health related quality of life in patients with actinic keratosis-an observational study of patients treated in dermatology specialist care in Denmark. Health Qual Life Outcomes 2015;13:111.

26. López-López D, Painceira-Villar R, Becerro-de-Bengoa-Vallejo $\mathrm{R}$, et al. Impact of the mechanical hyperkeratotic lesions and its association with quality of life: An observational case-control study. $J$ Eur Acad Dermatology Venereol 2018;32:1549-53.

27. Milobratović D, Janković S, Vukičević J, et al. Quality of life in patients with toenail onychomycosis. Mycoses 2013;56:543-51. 